\title{
Further Improve Circuit Partitioning Using GBAW Logic Perturbation Techniques
}

\author{
Yu-Liang Wu, Member, IEEE, Chak-Chung Cheung, David Ihsin Cheng, and Hongbing Fan
}

\begin{abstract}
Efficient circuit partitioning is becoming more and more important as the size of modern circuits keeps increasing. Conventionally, circuit partitioning is solved without altering the circuit by modeling the circuit as a hypergraph for the ease of applying graph algorithms. However, there is room for further improvement on even optimal hypergraph partitioning results, if logic information can be applied for circuit perturbation. Such logic transformation based partitioning techniques are relatively less addressed. In this paper, we present a powerful multiway partitioning technique which applies efficient logic rewiring techniques for further improvement over already superior hypergraph partitioning results. The approach can integrate with any graph partitioner. We perform experiments on two-, three-, and four-way partitionings for MCNC benchmark circuits whose physical and logical information are both available. Our experimental results show that this partitioning approach is very powerful. For example, it can achieve a further $12.3 \%$ reduction in cut size upon already excellent pure graph partitioner (hMetis) results on two-way partitioning with an area penalty of only $0.34 \%$. The outperforming results demonstrate the usefulness of this new partitioning technique.
\end{abstract}

Index Terms-Alternative wiring, partitioning.

\section{INTRODUCTION}

$\mathbf{T}$ HE objective of circuit partitioning is to divide the circuit into subcircuits so that the size of each component is reasonable and the number of interconnect between the components is minimized. As design scale expands, partitioning becomes increasingly important to circuit design automation.

Traditionally, circuit partitioning is done by simply modeling the circuit as a graph (or hypergraph). Graph partitioning problems are known to be NP-hard [1]. A comprehensive survey [2] has presented the directions of partitioning. Commonly used partitioning algorithms can be categorized into three classes. The first class strictly abides by the modeling graph, with no attempt to change the graph. High quality results have been reported by several algorithms that include iterative improvement based [1], [3], [4], clustering based [5], and spectrum (eigenvector) based [6], [7]. The second class of algorithms may modify the graph through node replications [8]-[11].

Manuscript received April 6, 2001; revised June 8, 2002. This work was supported in part by the Hong Kong Research Grants Council under Grant CUHK4236/01E and in part by Direct Grant CUHK2050244.

Y. L. Wu and C.-C. Cheung are with the Department of Computer Science and Engineering, The Chinese University of Hong Kong, Hong Kong (e-mail: ylw@cse.cuhk.edu.hk; cccheung@cse.cuhk.edu.hk).

D. I. Cheng is with Faraday Technology, Milpitas, CA 95035 USA (e-mail: dcheng@faraday-usa.com).

H. Fan is with the Department of Computer Science, University of Victoria, Victoria, BC V8W 3P6, Canada (e-mail: hfan@csr.csc.uvic.ca).

Digital Object Identifier 10.1109/TVLSI.2003.812369
Improvement is achieved by sacrificing areas due to node replications. These two classes both perform the partitioning task on the graph without considering the logic function of the circuit. The third class [12]-[15] couples the graph domain (nodes and their connections) and logic domain (function perform by each node). The tradeoff of improving the partitioning results is the high computational cost [13], [14] and may only be applied to field-programmable gate array (FPGA) circuits [15].

Recently, many research studies on multilevel partitioning have been proposed [16]-[22]. The general idea behind multilevel partitioning is to first cluster the whole problem by efficient algorithms so as to reduce the size, then apply a wellknown graph-domain partitioner on the coarsened graph to get a high quality initial solution. The graph is then unclustered and a suitable partitioning refinement algorithm is applied in order to adjust the cut edge between partitions. The quality and the runtime by multilevel partitioning are very encouraging. In particular, Karypis and Kumar [22] propose a partitioner called hMetis-Kway. It first coarsens the hypergraph, then recursively bisects the graph into k parts, followed by uncoarsening the hypergraph with refinement algorithms. More recent research works [23]-[26], in comparison with hMetis-Kway, have shown that the solution by hMetis-Kway is such a high quality that the cut size cannot be further reduced greatly.

Alternative wiring (rewiring) is the technique of adding single or multiple redundant wires or gates to a circuit so that other wires or gates become redundant and thus removable. This logic-domain technique has been widely used for solving many logic-level and physical-level design problems [12], [27]-[31]. Circuit performance can be improved by removing a wire on the critical path and adding its alternative wire elsewhere. Circuit routability can also be improved by substituting an unroutable wire in the congested area by a routable alternative wire in other circuit parts. The cut size of a partition can be reduced by replacing the wires crossing the cut line.

Fig. 1 illustrates how rewiring can be used to further improve an already optimal partitioning obtained by a typical graph-domain partition algorithm. The global optimal partitioning in the graph domain, with a cut size of 3, is shown in Fig. 1(a). However, if we apply the logic-domain rewiring technique to replace a target wire (thick line) crossing the cut line by its alternative wire (dotted line), the cut size can be further reduced to 2 as shown in Fig. 1(b) without injecting area increase. From this example, we can see that rewiring can be applied to partitioning to further improve upon even the optimal solution in the graph-domain. Binding the logic-domain rewiring technique with an efficient graph-domain partitioning tool enables a larger flexibility for obtaining better results. The rewiring technique can be used 


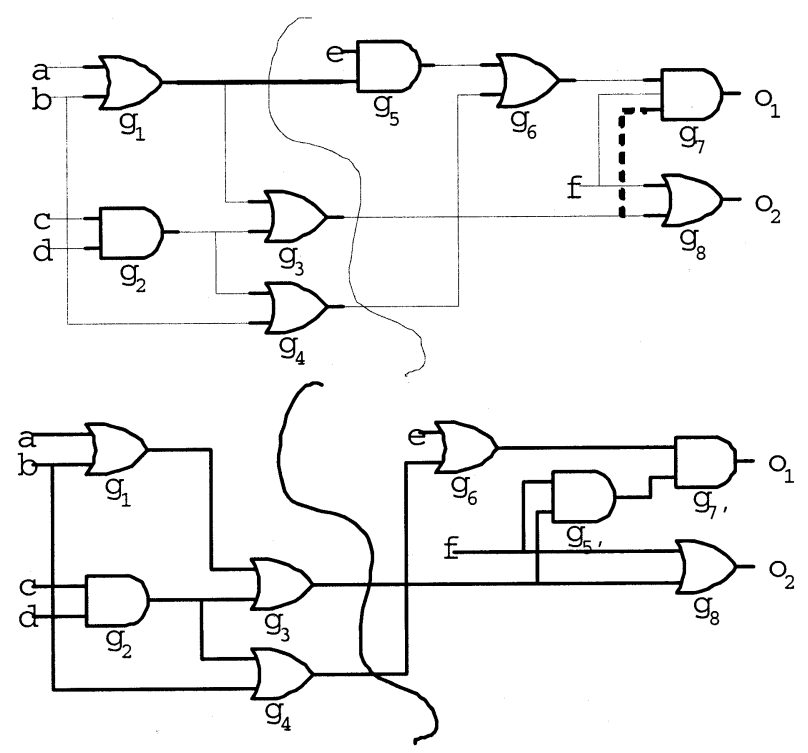

Fig. 1. Circuit partitioning by rewiring (cut net size improved from 3 to 2).

either as a greedily guided optimization tool, or as a random perturbation tool that allows for hill climbing on cut cost functions so as to release the cost out of local minima.

The well-known ATPG-based rewiring technique, redundancy addition and removal for multilevel Boolean optimization (RAMBO) [12], [14], [27], [28], [32]-[36], is a very powerful technique for identifying alternative wires of a specified target wire for a given circuit. This technique has been used for logic perturbations and has been integrated with a graph-domain partitioning algorithm to produce improved partitioning [12]. However, as it always selects a nonnegative-gain wire in replacing a target wire and it only considers simple cases of adding and replacing one single wire, it is easily trapped in local minima. Besides, the ATPG-based rewiring technique, though powerful, tends to spend much running time due to the time-consuming Boolean implication operations. Moreover, the benchmark circuits used in the Cheng's work [12] have been preprocessed by the SIS package [37] and have mapped into two-input gates.

To investigate the possibility of perturbing the circuit without applying any Boolean operations, minimal circuit structures yielding rewiring patterns have been studied [38], [39]. Based on benchmark circuits, we observe that the nearest existing alternative wire is quite close to its target wire. Therefore, these minimal patterns tend to be small and repeatedly appear in a circuit. As a result, instead of applying the ATPG-based logic implications repeatedly to a same pattern, the graph-based alternative wire (GBAW) technique [38], [39] employs a more efficient graph pattern matching operation to locate alternative wires. The basic idea of GBAW is to match the subcircuit with "prespecified" patterns. Rewiring by GBAW can be done without applying any logic implication or redundancy check, hence it runs very fast. Besides considering the alternative wire that is close to the target wire from those small "prespecified" patterns, distant alternative wires can also be located by propagating the matchings in a cascading way. By coupling RAMBO and GBAW as the perturbation engine, $\mathrm{Wu}$ [40] proposed the bipartitioning tool RAMBO-GBAW partitioner (RG) that also handles the two-input gates and has a larger flexibility for perturbation.

In our approach, excellent partitionings were firstly obtained using the pure graph-domain partitioner hMetis-Kway to serve as initial partitions. Then to expand the optimization space, we applied an iterative optimization process coupling both graph and logic domain partitioners. In graph domain, we chose the Fiduccia-Mattheyses (FM) partitioning algorithm [3] for its simplicity. In logic domain, we applied either the RAMBO [12], GBAW engine [38], or augmented GBAW [39], as a greedily guided perturbation engine.

Please note that the graph partitioner used in this approach is not limited to any particular one, i.e., the logic perturbation process can be coupled with any later developed more powerful graph-domain partitioning tool. We experimented this partition flow for two-, three- and four-way partitionings on various MCNC benchmarks ranging from small to fairly large circuits whose physical and logical information are both available. The results show that such a graph-logic domain coupled partitioning approach can further cut down the cut size effectively with small CPU and area overhead. Our results show that our proposed approach can further reduce the cut cost over excellent graph partitioner results by $12.3 \%, 11.1 \%$, and $11.4 \%$ for two- to four-way partitionings with quite low area overhead of $0.34 \%, 0.49 \%$, and $0.57 \%$ only, respectively. We observed that the experimental results amongst the three different logic perturbation engines are all significant and comparable, while the GBAW rewiring engine is the fastest one. The results seem to suggest that for partitioning objective, a simple rewiring scheme would be effective enough to produce the near best results. The encouraging results also suggest a quite promising approach for doing circuit partitioning.

This paper is organized as follows. The preliminaries and notion of alternative wiring are introduced in Section II. In Section III, a brief introduction to GBAW technique is given. In Section IV, the details of repartitioning by rewiring are shown. In Section V, experimental results are presented. Conclusions are drawn in Section VI.

\section{PRELIMINARIES}

A combinational circuit can be represented by a directed acyclic graph (DAG) where vertices correspond to the primary inputs (PI), primary outputs (PO) and the internal gates of the circuit. PI and PO are nodes that have only outgoing edges and incoming edges, respectively. An internal node has at least two incoming edges and one outgoing edge and is associated with a Boolean function. Inverters are not considered as internal nodes, but as polarity of edges during logic-domain perturbation. A Boolean network $G$ is used to represent a system of Boolean functions with specified variables as PI and functions as PO. The functionality of a Boolean network is specified by its primary output function set. Two Boolean networks are equivalent if they have the same functionality.

A wire is defined as a two-point connection between a pair of source and sink nodes. When a larger circuit is partitioned into two subcircuits, we define the wires crossing the partitioning 


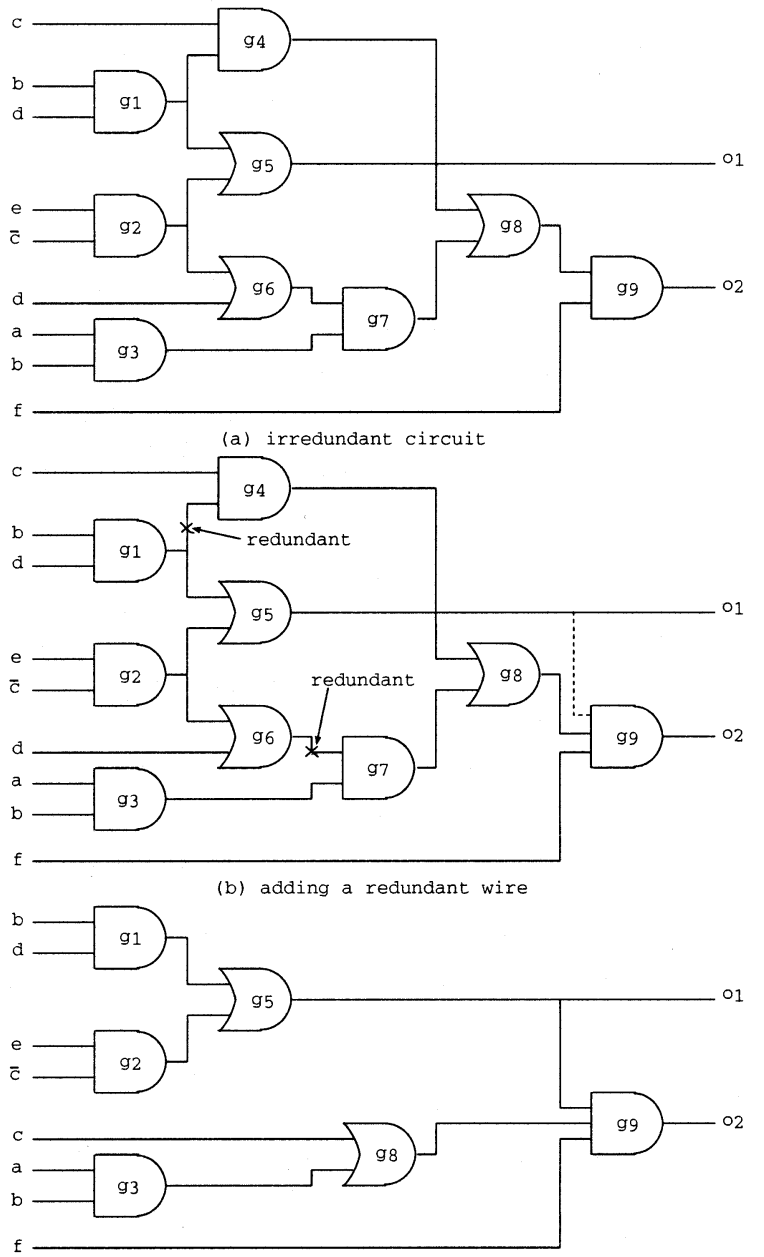

(c) final irredundant circuit

Fig. 2. Example of alternative wiring.

cut line as cut wires. We also define a cut net as a hyperedge connecting partitions and the cut pins as the total number of pins required for all partitioned blocks.

\section{A. Rewiring}

If we consider the circuit from Perturb and Simplify [28] as shown in Fig. 2(a), this circuit is irredundant because none of the wires in the circuit is removable. If we add a connection from the output of gate $g_{5}$ to the input of gate $g_{9}$ [shown as a dotted line in Fig. 2(b)], the functionality of the circuit does not change. In other words, the added connection is redundant. However, the addition of this connection causes two originally irredundant wires to become redundant as shown in Fig. 2(b). After removing these two wires and associated gates that either become floating $\left(g_{6}\right)$ or have a single fanin $\left(g_{4}\right.$ or $\left.g_{7}\right)$, the circuit can be greatly minimized as shown in Fig. 2(c). We can apply this rewiring techique to solve the logic synthesis and physical design problems.

\section{GBAW TECHNIQUE}

A wire is replaceable if it has at least one alternative wire. We use a graph configuration $D$ to represent a subnetwork function $S$ in a Boolean network $G$. In a Boolean network, the in-degree

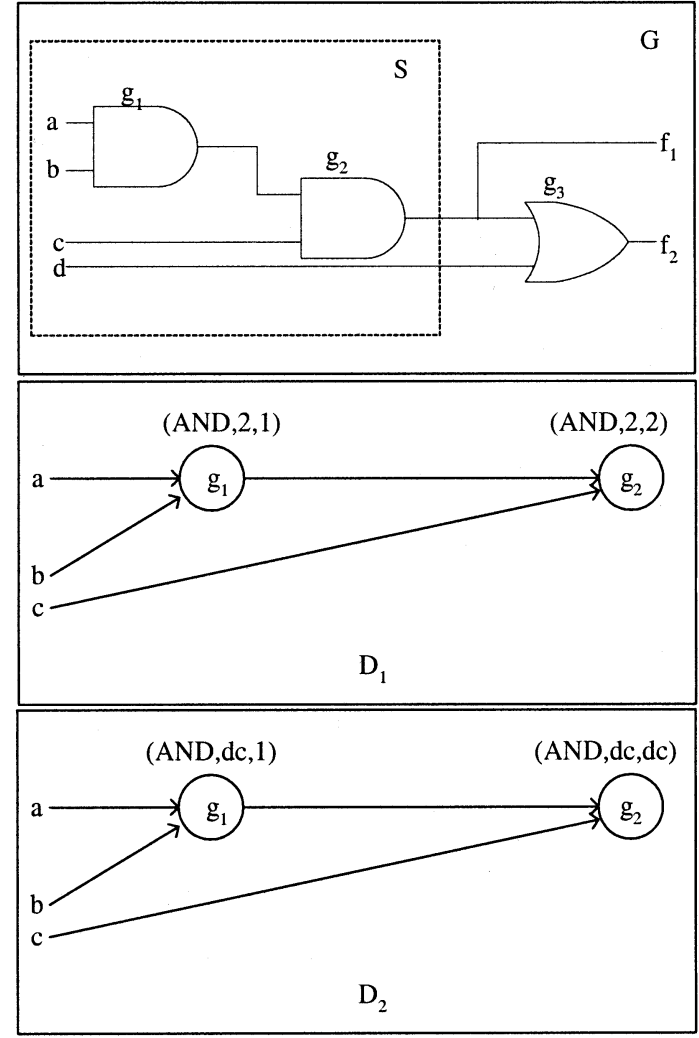

Fig. 3. Configuration of a subnetwork.

of node $y$, denoted by $d^{-}(y)$, is defined as the number of edges entering $y$. The out-degree of node $y$, denoted by $d^{+}(y)$, is defined as the number of edges leaving $y$. We also define a node $y$ by a triplet $\left(o p, d^{-}(y), d^{+}(y)\right)$, where $o p$ is the Boolean operator of $y$ that can be any associative operator like AND, OR, NAND, or NOR.

For each node $n_{i}$ in subnetwork $S$ in network $G, n_{i}$ is mapped to a triplet $\left(o p, i_{1}, i_{2}\right)$ in $D$ where $o p$ denotes the operator representing the Boolean function of $n_{i}$ and $i_{1}, i_{2}$ are nonnegative integers. All edges inside $S$ are preserved, while the edges outside $S$ are omitted in $D$. In most cases, $i_{1}$ equals $d^{-}\left(n_{i}\right)$ and $i_{2}$ equals $d^{+}\left(n_{i}\right)$. The element of a triplet $\left(o p, d^{-}(y), d^{+}(y)\right)$ can also be "don't care" (dc). For the first element, "dc" means any operator. For the other elements, "dc" can be any positive integers. We use a configuration to denote a minimal pattern containing both the target (the wire to be replaced) and its alternative (the wire to be added) wire. A minimal pattern implies that all the edges or nodes associated with the pattern cannot be removed.

The mapping is illustrated in Fig. 3. $S$ is a subnetwork of G. $D_{1}$ and $D_{2}$ are two mappable configurations of $S$. They are both called subgraph as they are mapped from a subnetwork $S$. A subgraph is minimal if all of its nodes and edges are all essential (unremovable) to the graph. A $k$-local pattern denotes a minimal subgraph with the distance between the alternative wire and its target wire being $k$. The distance between two wires is defined as the difference of maximum path length from any primary input to each of the wires. In Fig. 4(a), gate $y 3$ can be reached from $y 1$ and $I 4$ and the maximum path length is defined as 2 . 

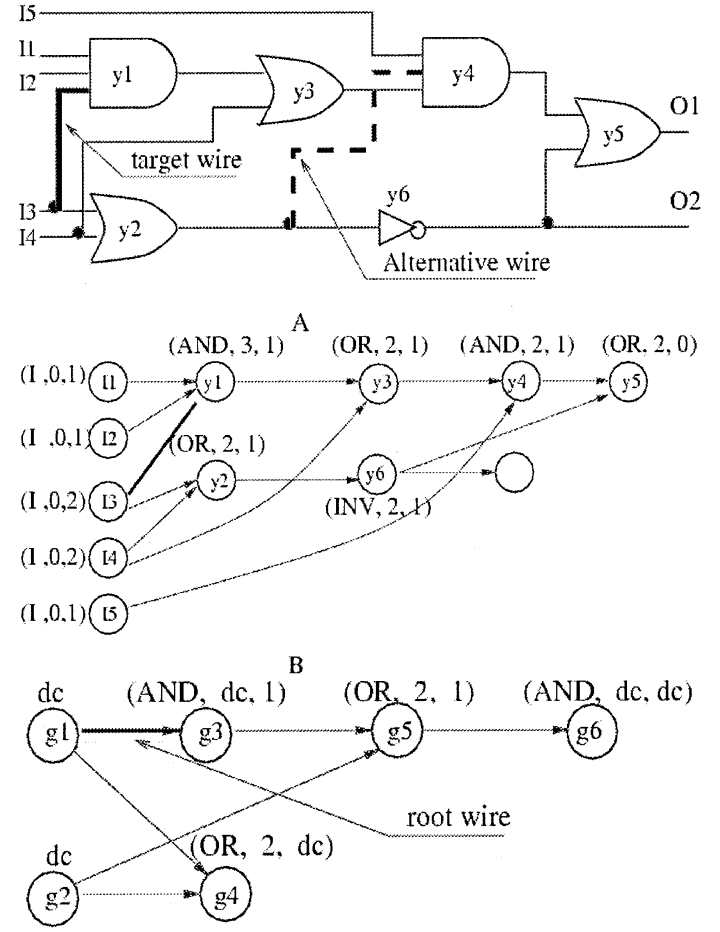

$\mathrm{C}=(\mathrm{D}, \mathrm{F})$

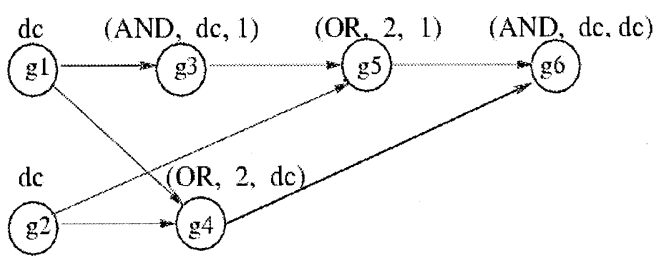

$C^{\prime}$

Fig. 4. A combinational network, a labeled Boolean network and two configurations.

Fig. 4 shows a combinational network (A) and its corresponding Boolean network (B) by using the configuration notation. We can further simplify this Boolean network into two configurations containing the alternative wire $\left(\mathrm{C}^{\prime}\right)$ and without containing redundant wire $(\mathrm{C})$. Finally, a pattern is constructed.

GBAW is a newly proposed and efficient rewiring technique. It models a circuit as a DAG and searches alternative wires by checking graph matchings between local subnetworks and the prespecified minimal subgraph configurations. A configuration is a minimal circuit pattern containing alternative wires within a given distance. Experiments show that the number of all such local minimal subgraphs is limited. Most of the alternative wires are located topologically "near" to their target wires. It has been shown that about $96 \%$ of the closest alternative wires are only two-edge distant from their target wires. When a subnetwork matches a pattern, GBAW can quickly determine the target wire and the corresponding alternative wires. Obviously, if $w_{r}$ is an alternative wire of $w_{t}$, then $w_{t}$ is also an alternative wire of $w_{r}$. Both $w_{t}$ and $w_{r}$ are pescribed in a pattern. But in a subnetwork, only one of them exists.

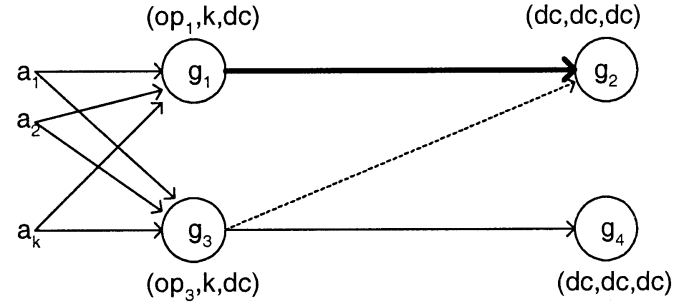

Fig. 5. 0-local pattern in GBAW.

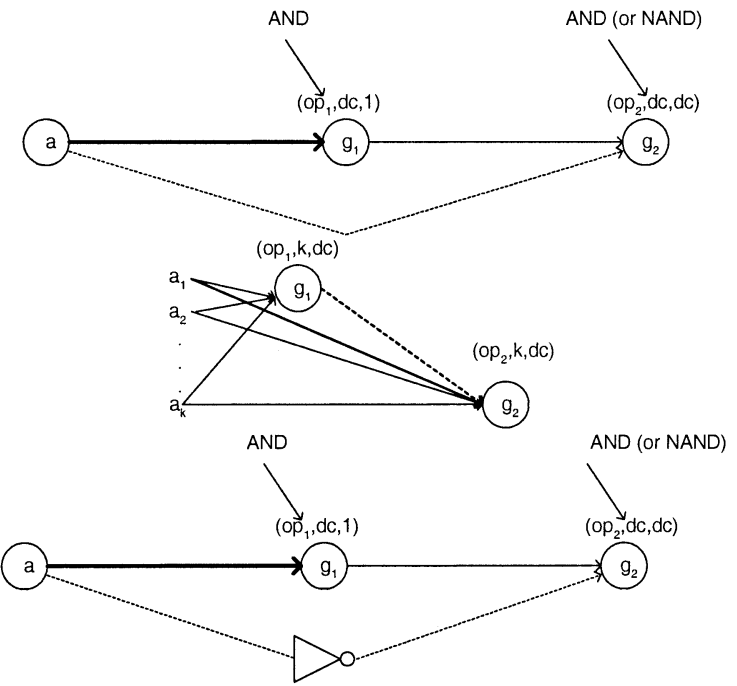

Fig. 6. 1-local patterns in GBAW.

\section{A. Alternative Wiring Patterns}

There are 0-local, 1-local, and 2-local patterns in GBAW and they are discussed briefly in the following subsections. According to our observations, the nearest alternative wire of a target wire is close to the target wire in most practical cases. In this paper, we apply an augmented GBAW scheme [39], which is a much extended scheme improved from GBAW version shown in $\mathrm{Wu}$ [38], to improve the effectiveness of identifying alternative wires of a given circuit for repartitioning. GBAW is able to find the alternative wire of the target wire within a limited distance; also it is able to locate a distant alternative wire by waveform propagations. This paper majorly applies GBAW as the perturbation engine in logic domain.

1) 0-Local Pattern: A 0-local pattern is a node substitution pattern such that two nodes can replace each other if they have the same logic function. As shown in Fig. 5, the target wire is $g_{1} \rightarrow g_{2}$ and its alternative wire is $g_{3} \rightarrow g_{2}$.

2) 1-Local Patterns: There are three basic types in 1-local patterns as shown in Fig. 6. Now, if we consider case 1-1, $a \rightarrow$ $g_{1}$ can be replaced by $a \rightarrow g_{2}$ if $o p_{1}=$ AND and $o p_{2}=$ AND or NAND. Case 1-2 can be proved by the following. Let $o p_{1}=$ NOR and $o p_{2}=$ AND, then $g_{1}=(a+x)^{\prime}=a^{*} x^{\prime}$, where $x=b_{1}+b_{2}+\cdots+b_{k}$ and $b_{1}, b_{2}, \ldots, b_{k}$ are the other inputs of $g_{1}$, or $x=0$ if $g_{1}$ has no other inputs. Then, $g_{2}=\left(g_{1}{ }^{*} y\right)$, where $y=c_{1}{ }^{*} c_{2}{ }^{*} \ldots{ }^{*} c_{l}$ and $c_{1}, c_{2}, \ldots, c_{l}$ are the other inputs of $g_{2}$, or $y=1$ if $g_{2}$ has no other inputs. Then $g_{2}$ can be regrouped as $g_{2}=\left(a^{\prime *} x^{\prime *} y\right)=\left(\left(x^{\prime}\right)^{*} a^{\prime *} y\right)=\left(g_{11} * a^{\prime *} y\right)$, where $g_{11}=x^{\prime}$ 


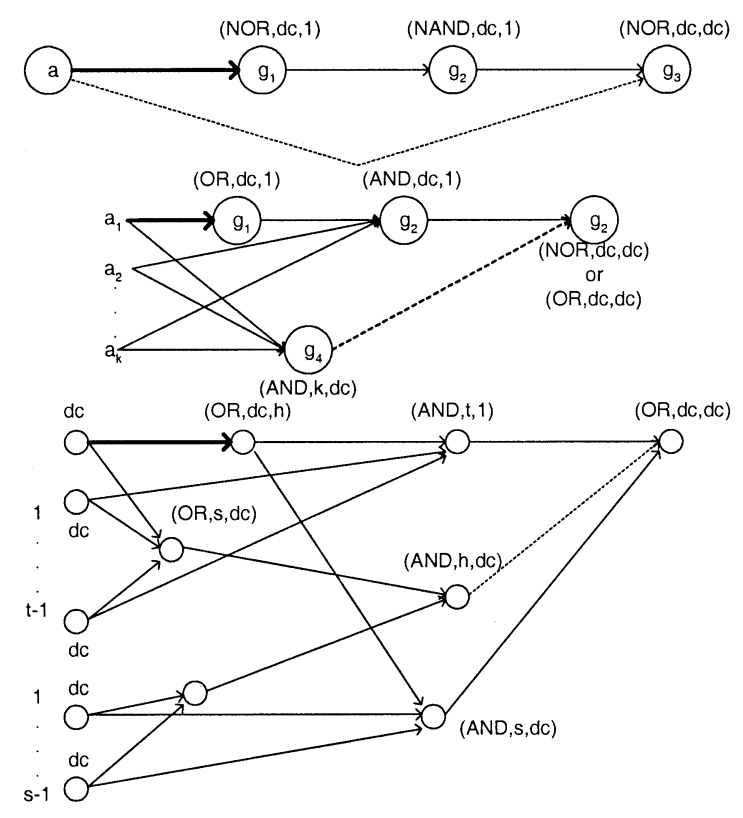

Fig. 7. 2-local patterns in GBAW.

is the logic function of $g_{1}$ after $a \rightarrow g_{1}$ is removed. Therefore, $a \rightarrow g_{2}$ is an alternative wire of $a \rightarrow g_{1}$.

3) 2-Local Patterns: In every 2-local patterns, the alternative wire is 2-edge far away from the target wire. In Fig. 7, three 2-local patterns are shown.

\section{B. Functional Analyses}

Fig. 8 shows four new 2-local patterns used in the augmented GBAW, with the target wire and its alternative wire shown as the thick line and dotted line respectively. The position of the target wire and alternative wire can be swapped. There are more than 40 different patterns in the implementation of augmented GBAW. GBAW does handle adding one wire and removing another one, adding one AND, OR, NAND, or NOR gate so as to remove one target wire. The patterns of the original GBAW are constructed based on basic minimal pattern configurations. In augmented GBAW, more patterns extracted from benchmark circuits using RAMBO tool are included.

\section{PARTITIONING Using Alternative WiRING}

The objective of a multiway partitioning is essentially to minimize the number of pins required to connect all partitions. Assume that one pin is used in a partition for a net. Since some of the wires may have alternative wires, if we replace cut wires by their alternative wires that are not cut wires, cut size can be reduced. The rewiring process may lead to new circuit graphs and in turn help escaping from local minima led by the graph domain partitioning process.

A rewiring perturbation refers to the replacement of a target wire by its alternative wires. Fig. 9 illustrates the gains regarding various perturbations in a circuit. In the figure, thick lines represent the target wires and dotted lines refer to their alternative wires. As shown in the example, we may have positive, zero, and negative gains.
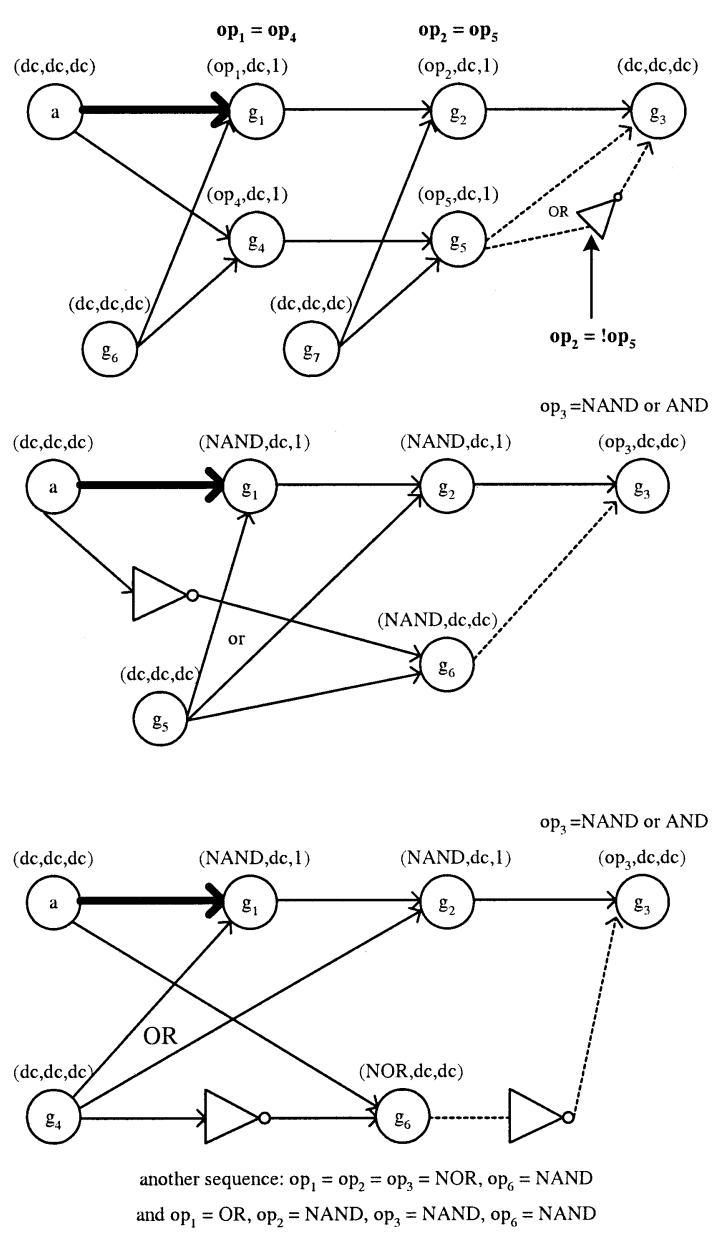

Fig. 8. Partial new 2-local patterns in GBAW.
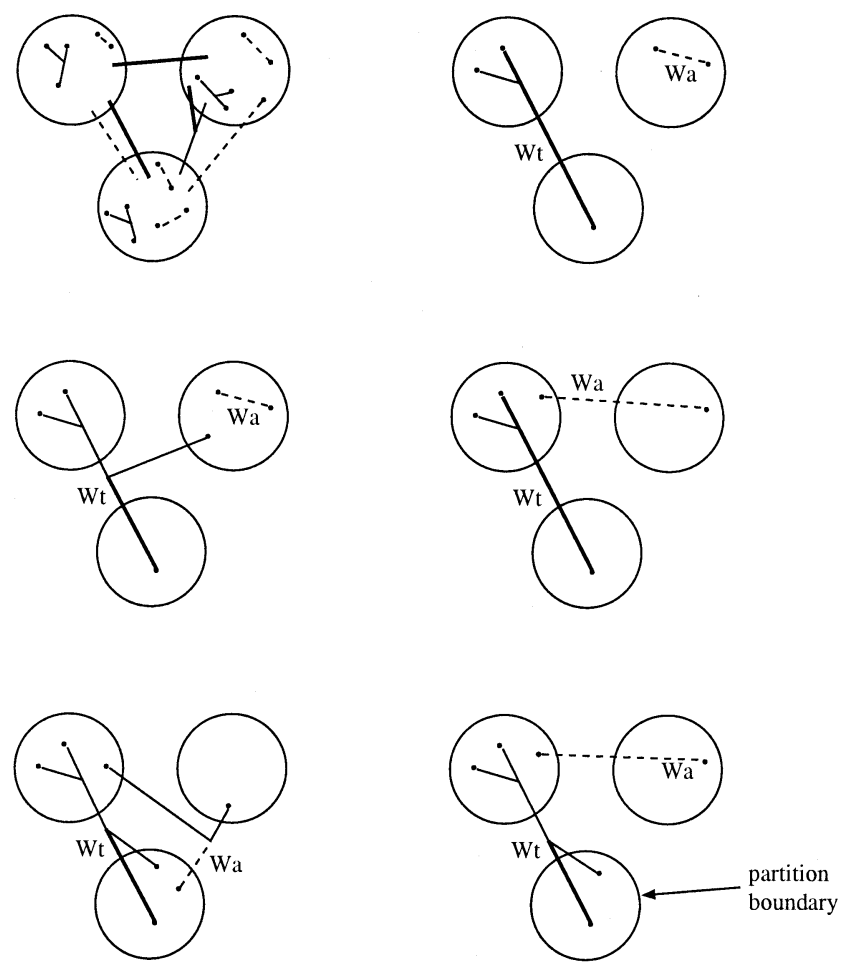

Fig. 9. Perturbations and cut pin gains for three-way partitioning. 


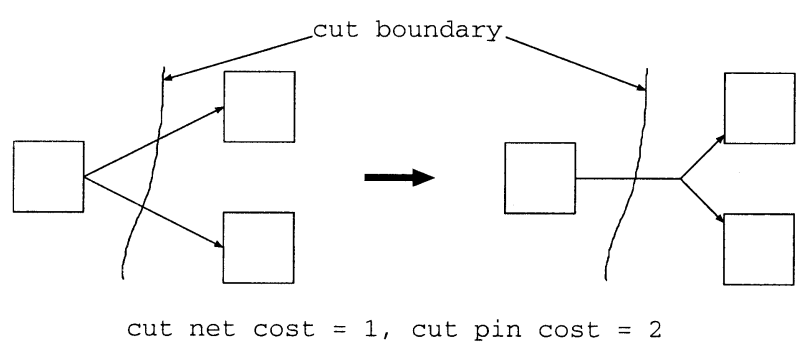

Fig. 10. Cut net cost versus cut pin cost in two-way partitioning.

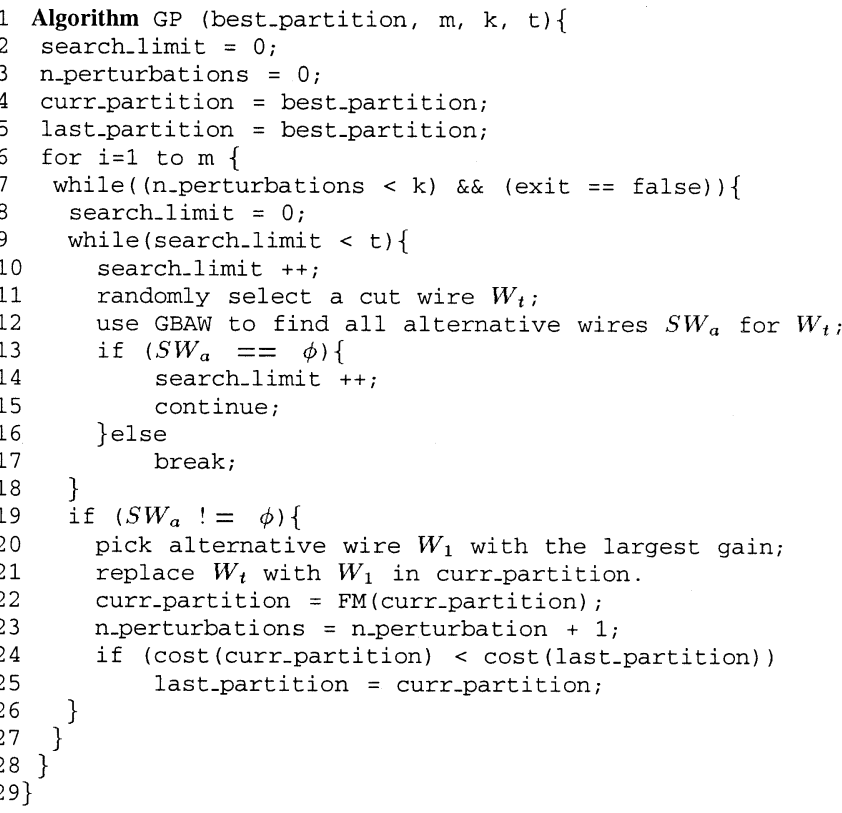

Fig. 11. Algorithm of GBAW-partitioner (GP).

As shown in Fig. 10, it shows the cut different between cut net cost and cut pin cost of a simple bipartition example. It is important to know that the cut net cost is only 1 but not 2 . It is inaccurate to measure the cut net cost in k-way partitioning, therefore, in our experiments we used the cut pin cost as the cut size in order to compare the quality of different partitioning approaches.

We use the hMetis-Kway partitioning tool to provide a fast and near optimal solution that serves as our initial partition. We adopt the well-known FM partitioning algorithm [3] as our graph-domain partitioner in our iterative graph-logic perturbation process for its simplicity and efficiency. In fact, we can apply any other graph domain partitioner for this purpose. Then we apply our rewiring technique (RAMBO for RP while GBAW for GP), to perform iterative logic perturbations aiming for further improvements. The perturbation operations include:

- substituting a wire with its alternative wire,

- adding a gate and removing several wires,

- adding one wire to remove other wires,

- adding two gates to remove other wires and so on.

Fig. 11 gives the algorithm of GP.

During the perturbation process GP, only cut wires will be selected as target wires for perturbations. We first randomly select a cut wire as the target wire. Then, GBAW is used to find the alternative wire set $S W a$ of the target wire. Finally, among the wire set $S W a$, the alternative wire with the highest gain

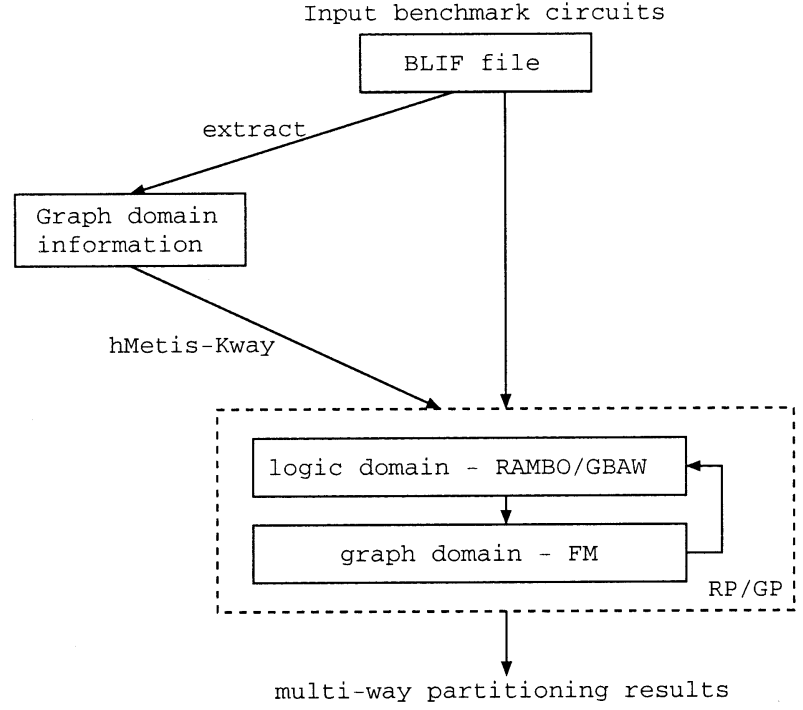

Fig. 12. Diagram shows the procedure of the experiments

is selected for perturbation. When the $S W a$ of the target cut wire is empty, GP may randomly select another cut wire for another trial. The number of iterations is set by $m$. The number of trials is limited by $t$ times. $k$ is the limit of perturbations. These limits serve to set bounds for unnecessary runs when the total number of alternative wires of all cut wires is zero or very small. $\mathrm{RP}$ is similar to GP except that RAMBO is used for rewiring. The main difference between algorithm GP and the algorithm in [12] lies in the condition of perturbations. In [12], a perturbation is performed only when the alternative wire of the selected cut wire has a nonnegative gain. However, in our experiments, hill-climbing perturbations are allowed, therefore the chance of obtaining better solutions can increase. In this paper, a negative-gain perturbation is also allowed to help escaping local minimums. On the other hand, the main difference between GP and the algorithm in $\mathrm{Wu}[40]$ is the perturbation engine used. In $\mathrm{Wu}$ [40], a coupling scheme of RAMBO and GBAW (RG) is used.

\section{EXPERIMENTAL RESULTS}

The algorithm GP was implemented in $\mathrm{C}$ and the experiments were conducted on Sun Enterprise E4500 workstation with 8 GB memory in a single-processor configuration for circuits of various sizes from MCNC benchmarks. The large benchmark circuits used in ISPD98 [41] are not applicable for our experiments due to the lack of logical-domain information. The benchmark circuits are first mapped into 2-input gates by using SIS [37] package. The logic minimization by SIS [37] standard script script.algebraic is conducted on each benchmark circuit in the preprocess step. Fig. 12 shows the precedure of the experiments.

There are 29 MCNC benchmark circuits in our experiments. The number of nodes and wires of each circuit are shown in Table I. Column "literals," "PI," and "PO" show the literal counts, the number of primary inputs, and the number of primary ouputs, respectively. This table also lists the statistics of alternative wires on the benchmark circuits and the results are separated into three parts which are RAMBO, GBAW, and the augmented GBAW. Column "Circuit" shows the name of the circuit. Column "alt. wire" lists the number of alternative 
TABLE I

BENCHMARK CIRCUITS STATISTICS

\begin{tabular}{|c|c|c|c|c|c|c|c|c|c|c|c|}
\hline \multirow[t]{2}{*}{ Circuit } & \multirow[t]{2}{*}{ Node } & \multirow[t]{2}{*}{ Wire } & \multirow[t]{2}{*}{ literals } & \multirow[t]{2}{*}{ PI } & \multirow[t]{2}{*}{$\mathrm{PO}$} & \multicolumn{2}{|c|}{ RAMBO } & \multicolumn{2}{|c|}{ GBAW } & \multicolumn{2}{|c|}{ Augmented GBAW } \\
\hline & & & & & & alt. wires & CPU & alt. wires & $\mathrm{CPU}$ & alt. wires & CPU \\
\hline $5 \times 1$ & 132 & 257 & 235 & 7 & 10 & 55 & 4.84 & 52 & 0.08 & 56 & 0.15 \\
\hline 9sym-hdl & 141 & 273 & 232 & 9 & 1 & 72 & 1.63 & 35 & 0.06 & 36 & 0.08 \\
\hline $\mathrm{C} 1355$ & 600 & 1159 & 1055 & 41 & 32 & 178 & 20.43 & 250 & 0.30 & 250 & 0.64 \\
\hline $\mathrm{C} 1908$ & 516 & 999 & 883 & 33 & 25 & 282 & 35.48 & 312 & 0.23 & 312 & 0.39 \\
\hline C2670 & 1043 & 1853 & 1444 & 233 & 140 & 471 & 81.54 & 502 & 0.43 & 528 & 0.91 \\
\hline C3540 & 1263 & 2476 & 2267 & 50 & 22 & 1039 & 289.79 & 1219 & 0.75 & 1228 & 1.49 \\
\hline $\mathrm{C} 432$ & 238 & 440 & 392 & 36 & 7 & 238 & 9.58 & 250 & 0.14 & 250 & 0.25 \\
\hline C499 & 503 & 965 & 854 & 41 & 32 & 32 & 10.74 & 34 & 0.22 & 34 & 0.33 \\
\hline C5315 & 1962 & 3746 & 3282 & 178 & 123 & 728 & 139.27 & 778 & 0.96 & 799 & 1.94 \\
\hline C6288 & 2856 & 5680 & 5195 & 32 & 32 & 2644 & 443.36 & 2214 & 1.54 & 2214 & 2.44 \\
\hline C7552 & 2422 & 4637 & 4105 & 207 & 108 & 902 & 285.54 & 680 & 1.81 & 734 & 2.83 \\
\hline $\mathrm{C} 880$ & 483 & 906 & 780 & 60 & 26 & 257 & 13.94 & 280 & 0.22 & 280 & 0.40 \\
\hline alu2 & 422 & 834 & 777 & 10 & 6 & 313 & 127.16 & 310 & 0.31 & 322 & 0.55 \\
\hline alu4 & 785 & 1556 & 1470 & 14 & 8 & 597 & 238.35 & 586 & 0.55 & 624 & 1.04 \\
\hline apex6 & 908 & 1681 & 1417 & 135 & 99 & 412 & 46.41 & 474 & 0.46 & 487 & 0.82 \\
\hline b9_n2 & 157 & 273 & 208 & 41 & 21 & 62 & 1.60 & 77 & 0.06 & 78 & 0.11 \\
\hline comp & 184 & 336 & 270 & 32 & 3 & 106 & 4.56 & 88 & 0.08 & 108 & 0.14 \\
\hline des & 3839 & 7422 & 6655 & 256 & 245 & 2643 & 1128.52 & 3334 & 4.81 & 3336 & 6.56 \\
\hline duke2 & 386 & 750 & 676 & 22 & 29 & 264 & 47.07 & 316 & 0.24 & 318 & 0.42 \\
\hline $\mathrm{f} 51 \mathrm{~m}$ & 137 & 266 & 244 & 8 & 8 & 59 & 6.10 & 63 & 0.08 & 65 & 0.15 \\
\hline $\operatorname{misex} 3$ & 538 & 1062 & 990 & 14 & 14 & 414 & 125.51 & 507 & 0.39 & 507 & 0.65 \\
\hline my adder & 212 & 391 & 339 & 33 & 17 & 48 & 1.65 & 0 & 0.07 & 0 & 0.11 \\
\hline pcler8 & 130 & 233 & 174 & 27 & 17 & 30 & 1.57 & 30 & 0.03 & 42 & 0.10 \\
\hline rot & 824 & 1513 & 1251 & 135 & 107 & 402 & 32.91 & 435 & 0.37 & 443 & 0.69 \\
\hline sao2-hdl & 250 & 490 & 439 & 10 & 4 & 209 & 16.57 & 171 & 0.12 & 171 & 0.28 \\
\hline term 1 & 272 & 510 & 439 & 34 & 10 & 191 & 11.97 & 197 & 0.12 & 198 & 0.24 \\
\hline $\mathrm{tt} 2$ & 227 & 430 & 376 & 24 & 21 & 156 & 5.89 & 144 & 0.13 & 149 & 0.21 \\
\hline $\mathrm{x} 3$ & 855 & 1575 & 1334 & 135 & 99 & 385 & 33.63 & 453 & 0.43 & 459 & 0.79 \\
\hline Total & & & & & & 13189 & 3165.61 & 13791 & 14.99 & 14028 & 24.71 \\
\hline Normalized & & & & & & 1 & 1 & 1.04 & $0.47 \%$ & 1.06 & $0.78 \%$ \\
\hline
\end{tabular}

TABLE II

Comparison of 2-WAy Partitioning BetweEn hMetis-Kway \& RP AUGMENTED GP \& GP

\begin{tabular}{|c|c|c|c|c|c|c|c|c|c|c|c|c|c|c|c|c|}
\hline \multirow[t]{2}{*}{ Circuit } & \multicolumn{4}{|c|}{ * hMetis-Kway (Initial Part.) } & \multicolumn{4}{|c|}{ RAMBO-Partitioner } & \multicolumn{4}{|c|}{ Augmented-GBAW Partitioner } & \multicolumn{4}{|c|}{ GBAW-Partitioner } \\
\hline & area & \#lits & $\begin{array}{l}\text { cut } \\
\text { pins }\end{array}$ & $\star$ сри & area & \#lits & $\begin{array}{l}\text { cut } \\
\text { pins }\end{array}$ & cpu & area & \#lits & $\begin{array}{l}\text { cut } \\
\text { pins }\end{array}$ & сpu & area & \#lits & $\begin{array}{l}\text { cut } \\
\text { pins } \\
\end{array}$ & cpu \\
\hline $5 \times \mathrm{xpl}$ & $61: 71$ & 235 & 30 & 90 & $71: 63$ & 237 & 36 & 3 & 59:79 & 241 & 30 & 2 & $75: 60$ & 238 & 28 & 1 \\
\hline 9sym-hdl & $67: 74$ & 232 & 16 & 103 & $67: 74$ & 232 & 16 & 0 & $74: 67$ & 232 & 16 & 0 & $67: 74$ & 232 & 16 & 0 \\
\hline $\mathrm{C} 1355$ & $272: 328$ & 1055 & 48 & 203 & $324: 281$ & 1060 & 36 & 4 & $334: 272$ & 1061 & 40 & 3 & $328: 273$ & 1056 & 36 & 3 \\
\hline C1908 & $268: 248$ & 883 & 82 & 193 & $254: 271$ & 892 & 68 & 27 & 270:250 & 887 & 64 & 13 & 253:264 & 883 & 64 & 11 \\
\hline C2670 & $513: 530$ & 1444 & 42 & 250 & $520: 535$ & 1456 & 36 & 26 & $525: 525$ & 1451 & 34 & 26 & $513: 535$ & 1449 & 34 & 20 \\
\hline C 3540 & 632:631 & 2267 & 134 & 615 & 615:664 & 2283 & 120 & 38 & $617: 656$ & 2276 & 114 & 43 & 623:650 & 2276 & 116 & 34 \\
\hline $\mathrm{C} 432$ & 119:119 & 392 & 44 & 133 & 124:123 & 401 & 36 & 21 & 119:129 & 402 & 36 & 6 & 129:119 & 402 & 36 & 5 \\
\hline C499 & $231: 272$ & 854 & 48 & 153 & $272: 236$ & 859 & 36 & 5 & $237: 267$ & 855 & 36 & 2 & $272: 232$ & 855 & 36 & 2 \\
\hline C5315 & 1046:916 & 3282 & 104 & 665 & 1053:918 & 3291 & 100 & 14 & $1047: 91$ & 3286 & 100 & 57 & 1047:919 & 3286 & 100 & 44 \\
\hline C6288 & $1455: 1312$ & 5195 & 82 & 813 & 1296: 1575 & 5210 & 78 & 22 & $1317: 1545$ & 5201 & 78 & 111 & $1295: 1573$ & 5207 & 78 & 98 \\
\hline C7552 & 1281:1141 & 4105 & 18 & 670 & 1147:1281 & 4111 & 18 & 75 & $1286: 1142$ & 4111 & 18 & 77 & 1142:1286 & 4111 & 18 & 57 \\
\hline C880 & $261: 222$ & 780 & 50 & 173 & $259: 235$ & 791 & 36 & 8 & $249: 245$ & 789 & 36 & 13 & $262: 233$ & 789 & 36 & 11 \\
\hline alu2 & $190: 232$ & 777 & 82 & 235 & $197: 255$ & 807 & 80 & 64 & $175: 252$ & 781 & 78 & 15 & $179: 251$ & 785 & 78 & 11 \\
\hline alu4 & $431: 354$ & 1470 & 140 & 368 & 371:444 & 1500 & 128 & 66 & 434:363 & 1482 & 118 & 27 & $353: 446$ & 1484 & 120 & 20 \\
\hline apex6 & $435: 473$ & 1417 & 18 & 198 & $435: 473$ & 1417 & 18 & 3 & $435: 473$ & 1417 & 18 & 1 & $435: 473$ & 1417 & 18 & 1 \\
\hline b9_-122 & $87: 70$ & 208 & 16 & 95 & $65: 93$ & 209 & 12 & 0 & 63:94 & 208 & 12 & 0 & $65: 92$ & 208 & 12 & 0 \\
\hline comp & 93:91 & 270 & 6 & 98 & $91: 92$ & 269 & 8 & 1 & 90:93 & 269 & 6 & 4 & 90:93 & 269 & 6 & 3 \\
\hline des & 1894:1945 & 6655 & 286 & 1370 & 1989:1939 & 6744 & 276 & 34 & $1606: 2233$ & 6655 & 246 & 173 & 2074:1805 & 6684 & 246 & 150 \\
\hline duke2 & 179:207 & 676 & 82 & 210 & $232: 177$ & 699 & 76 & 10 & $160: 231$ & 680 & 78 & 12 & $167: 228$ & 684 & 74 & 9 \\
\hline $\mathrm{f} 5 \mathrm{~lm}$ & $72: 65$ & 244 & 28 & 125 & $81: 64$ & 252 & 28 & 8 & 64:76 & 247 & 28 & 1 & $76: 64$ & 247 & 28 & 1 \\
\hline misex 3 & 293:245 & 990 & 80 & 265 & 250:314 & 1016 & 72 & 48 & 249:301 & 1002 & 72 & 18 & $302: 250$ & 1004 & 76 & 14 \\
\hline my_adder & $106: 106$ & 339 & 4 & 115 & 106: 106 & 339 & 4 & 0 & 106:106 & 339 & 4 & 0 & 106:106 & 339 & 4 & 0 \\
\hline pcler8 & $58: 72$ & 174 & 8 & 75 & $61: 72$ & 177 & 8 & 3 & $66: 65$ & 175 & 8 & 0 & $58: 72$ & 174 & 8 & 0 \\
\hline rot & 441:383 & 1251 & 56 & 273 & $399: 431$ & 1257 & 52 & 12 & $384: 441$ & 1252 & 48 & 22 & $399: 427$ & 1253 & 48 & 17 \\
\hline sao2-hdl & $136: 114$ & 439 & 26 & 140 & $114: 136$ & 418 & 26 & 0 & $128: 123$ & 440 & 16 & 3 & $129: 122$ & 440 & 16 & 2 \\
\hline terml & $124: 148$ & 439 & 28 & 155 & $159: 130$ & 456 & 24 & 5 & 131:149 & 447 & 24 & 7 & 149:130 & 446 & 24 & 5 \\
\hline too_large & $2161: 1913$ & 6387 & 340 & 140 & 1762:1702 & 6475 & 312 & 13825 & 1714:1664 & 6390 & 312 & 245 & $1745: 1631$ & 6388 & 312 & 219 \\
\hline $\mathrm{ttt} 2$ & $120: 107$ & 376 & 10 & 98 & 120:107 & 376 & 12 & 0 & $107: 120$ & 376 & 10 & 0 & 120:107 & 376 & 8 & 0 \\
\hline $\mathrm{x} 3$ & 471:384 & 1334 & 22 & 210 & 470:389 & 1338 & 12 & 18 & $469: 389$ & 1337 & 16 & 6 & 470:389 & 1338 & 16 & 5 \\
\hline Total & & 44170 & 1930 & & & 44572 & 1764 & 14340 & & 44289 & 1696 & 887 & & 44320 & 1692 & 743 \\
\hline Average & & & & & & $+0.91 \%$ & $-8.6 \%$ & 19.30 & & $+0.27 \%$ & $-12.1 \%$ & 1.19 & & $+0.34 \%$ & $-12.3 \%$ & 1 \\
\hline
\end{tabular}

* (The result was picked from 250 runs of hMetis-Kway)

* (Total CPU times of 250 runs of hMetis-Kway)

cut size: the total number of pins required for all partitioned blocks

wires found in the circuits. Column "CPU" refers to the runtime in seconds. From the experimental results listed in Table I, we observe that GBAW and RAMBO have comparable AW searching power while GBAW uses barely $1 \%$ CPU usage of RAMBO. The augmented GBAW includes many newly formed 2-local patterns, which leads to better capability in locating
AWs than the original GBAW engine.We give an example on how GP works below. Taking a C3540 benchmark as an example, the circuit is first partitioned by hMetis-Kway for 250 times to obtain an initial partition with cut pin cost of 134. The GP algorithm is then applied to further cut down the cost to 116 through the following steps. 
TABLE III

COMPARISON OF THREe-WAY PARTITIONING BETWEEN HMETIS-KwAY ANDGBAW-PARTITIONER

\begin{tabular}{|c|c|c|c|c|c|c|c|c|}
\hline \multirow[t]{2}{*}{ Circuit } & \multicolumn{4}{|c|}{ * hMetis-Kway (Initial Partitioning) } & \multicolumn{4}{|c|}{ GBAW-Partitioner } \\
\hline & area & \#lits & $\begin{array}{l}\text { cut } \\
\text { pins }\end{array}$ & $\star \mathrm{cpu}$ & area & \#lits & $\begin{array}{l}\text { cut } \\
\text { pins }\end{array}$ & срu \\
\hline $5 \times 1$ & $37: 43: 52$ & 235 & 53 & 167 & $41: 44: 52$ & 240 & 50 & 1 \\
\hline 9sym-hdl & $43: 44: 54$ & 232 & 32 & 168 & $43: 44: 55$ & 232 & 22 & 0 \\
\hline C1355 & 217:196:187 & 1055 & 84 & 298 & 217:201:190 & 1064 & 82 & 7 \\
\hline C1908 & 148:186:182 & 883 & 116 & 275 & 146:167:205 & 886 & 101 & 6 \\
\hline $\mathrm{C} 2670$ & $354: 325: 364$ & 1444 & 82 & 335 & $358: 310: 375$ & 1445 & 70 & 11 \\
\hline C3540 & 378:428:457 & 2267 & 185 & 720 & 376:485:404 & 2286 & 176 & 18 \\
\hline $\mathrm{C} 432$ & $67: 79: 92$ & 392 & 56 & 168 & $70: 95: 77$ & 405 & 54 & 3 \\
\hline $\mathrm{C} 499$ & $143: 187: 173$ & 854 & 78 & 210 & 142:170:192 & 856 & 67 & 2 \\
\hline C5315 & 581:705:676 & 3282 & 119 & 828 & 522:704:741 & 3289 & 103 & 25 \\
\hline C6288 & $823: 954: 1079$ & 5195 & 150 & 995 & $814: 1110: 934$ & 5208 & 145 & 57 \\
\hline C7552 & 697:814:911 & 4105 & 91 & 855 & 699:943:782 & 4114 & 76 & 35 \\
\hline C880 & $142: 159: 182$ & 780 & 72 & 265 & $147: 168: 174$ & 790 & 58 & 6 \\
\hline alu2 & $121: 164: 137$ & 777 & 139 & 308 & 117:139:168 & 793 & 128 & 6 \\
\hline alu4 & 229:248:308 & 1470 & 209 & 515 & 218:313:255 & 1483 & 195 & 12 \\
\hline apex6 & $276: 347: 285$ & 1417 & 75 & 398 & $257: 355: 303$ & 1431 & 72 & 10 \\
\hline b9_n2 & $45: 59: 53$ & 208 & 21 & 128 & $44: 60: 54$ & 209 & 21 & 0 \\
\hline comp & $57: 65: 62$ & 270 & 16 & 148 & $57: 62: 66$ & 271 & 14 & 1 \\
\hline des & $1093: 1514: 1232$ & 6655 & 350 & 1765 & $1044: 1261: 1535$ & 6658 & 248 & 87 \\
\hline duke2 & $112: 124: 150$ & 676 & 130 & 295 & 107:135:144 & 691 & 115 & 5 \\
\hline $\mathrm{f} 51 \mathrm{~m}$ & $41: 53: 43$ & 244 & 58 & 170 & $56: 48: 38$ & 248 & 50 & 2 \\
\hline $\operatorname{misex} 3$ & $153: 209: 176$ & 990 & 126 & 375 & $155: 209: 175$ & 1004 & 117 & 7 \\
\hline my_adder & $80: 65: 67$ & 339 & 8 & 150 & 67:78:67 & 339 & 8 & 0 \\
\hline pcler8 & $41: 40: 49$ & 174 & 17 & 118 & $41: 40: 49$ & 174 & 17 & 0 \\
\hline rot & $235: 324: 265$ & 1251 & 83 & 360 & $237: 329: 259$ & 1255 & 74 & 9 \\
\hline sao2-hdl & $94: 77: 79$ & 439 & 70 & 215 & $91: 76: 86$ & 446 & 60 & 3 \\
\hline term1 & $77: 106: 89$ & 439 & 54 & 220 & $78: 108: 89$ & 442 & 50 & 3 \\
\hline too_large & $958: 1330: 1087$ & 6387 & 749 & 2615 & $970: 1058: 1351$ & 6395 & 683 & 114 \\
\hline $\mathrm{tt} 2$ & $64: 74: 89$ & 376 & 33 & 195 & $66: 89: 75$ & 379 & 33 & 2 \\
\hline $\mathrm{x} 3$ & 243:277:335 & 1334 & 78 & 345 & $237: 283: 340$ & 1354 & 75 & 10 \\
\hline $\begin{array}{c}\text { Total } \\
\text { Average }\end{array}$ & & 44170 & 3334 & & & $\begin{array}{c}44387 \\
+0.49 \%\end{array}$ & $\begin{array}{c}2964 \\
-11.1 \%\end{array}$ & \\
\hline
\end{tabular}

* (The result was picked from 250 runs of hMetis-Kway)

* (Total CPU times of 250 runs of hMetis-Kway)

cut size: the total number of pins required for all partitioned blocks

- GP searches all the alternative wires of the wires which lie along the cut line and replaces them and the original graph is changed with gain 5 .

- With a logically equivalent but different graph, FM reduced the cost down to 122 .

- Again, GP searches along the cut line and reduces the total cost by 6 .

- By switching between graph and logic domain, the cost is reduced to 116 .

In our experiments, we set the tolerance of area imbalance of $\mathrm{RP} / \mathrm{GP}$ to be $\pm 20 \%$ of the average area in each partitioned block. Therefore, the maximal ratios are $40 \%: 60 \%$ and $20 \%: 30 \%$ in two-way and four-way partitionings, respectively. The graphdomain partitioner of RP/GP is FM and the logic-domain partitioner is RAMBO/GBAW. In order to obtain excellent initial partitions, hMetis-Kway [22] was run 250 times to pick the best result for each circuit. As hMetis-Kway is known to be quite powerful, the initial partioning results should be very excellent if not near optimum. The next step is to apply RP/GP for logic perturbation to further improve the high quality graph partitioning results with the setting of $k=10$ and $t=50$. Table II lists the experimental results for the two-way partitionings by four different approaches. The first one is the initial partitioning by hMetis-Kay, the second one is the ATPG-based RAMBO partitioner (RP), the third one is the augmented GBAW partitioner (GP2), and the last one, the GBAW partitioner (GP). Column "area" lists the area of the subcircuit in terms of the number of gates. "\#lits" lists the total number of literals of the partitioned circuits, which is used to measure the size of the circuit. From the results, the area penalties for two-way partitionings by RP, GP2, and GP are $0.91 \%, 0.21 \%$, and $0.34 \%$, respectively. Column "cut pins" lists the total number of pins required for all partitioned blocks, which should be double of the cut net size in a two-way partitioning. Column "cpu" lists the cpu time (in seconds).

We can see that applying logic perturbation can further reduce the cut size of the good partitionings produced by the purely graph-domain partitioner significantly. The total number of literals is slightly increased because of the added gates during perturbations. Table II shows that the approach can obtain $8.6 \%$, $12.1 \%$, and $12.3 \%$ further reduction on cut size over the already excellent hMetis-Kway cut results in two-way partitionings using RP, GP2, and GP, respectively.

On average, RP took nearly 20 times of CPU usage than GP. In GP2, the logic-domain engine is able to locate more 2-local patterns than GP while the cut reduction is similar. Either rewiring engine applied in the logic domain can always produce further significant cut size reduction upon graph partitioner results. As GP is the fastest engine amongst the three partitioners while produces near best results, we give experimental results on three-way and four-way partitioning by using GP only in Tables III and IV. We obtained $11.1 \%$ and $11.4 \%$ reduction in cut pins for the three-way and four-way partitionings with area penalties of $0.49 \%$ and $0.57 \%$ only, respectively. 
TABLE IV

COMPARISON OF 4-WAY PARTITIONING BETWEEN HMETIS-KWAY \& GBAW-PARTITIONER

\begin{tabular}{|c|c|c|c|c|c|c|c|c|}
\hline \multirow[t]{2}{*}{ Circuit } & \multicolumn{4}{|c|}{ * hMetis-Kway (Initial Partitioning) } & \multicolumn{4}{|c|}{ GBAW-Partitioner } \\
\hline & area & \#lits & $\begin{array}{c}\text { cut } \\
\text { pins }\end{array}$ & $\star \mathrm{cpu}$ & area & \#lits & $\begin{array}{c}\text { cut } \\
\text { pins }\end{array}$ & cpu \\
\hline$\overline{5 x p 1}$ & $40: 31: 34: 27$ & 235 & 67 & 170 & $39: 31: 30: 37$ & 239 & 61 & 1 \\
\hline 9sym-hdl & $38: 36: 36: 31$ & 232 & 48 & 200 & $28: 35: 37: 40$ & 231 & 26 & 0 \\
\hline C1355 & 141:130:177:152 & 1055 & 102 & 398 & 154:180:137:137 & 1077 & 99 & 8 \\
\hline $\mathrm{C} 1908$ & 130:131:116:139 & 883 & 134 & 382 & $125: 137: 118: 136$ & 886 & 109 & 6 \\
\hline $\mathrm{C} 2670$ & $289: 238: 263: 253$ & 1444 & 124 & 438 & $289: 252: 240: 265$ & 1453 & 84 & 11 \\
\hline C3540 & 281:328:357:297 & 2267 & 252 & 840 & $274: 354: 356: 281$ & 2280 & 219 & 22 \\
\hline $\mathrm{C} 432$ & 64:57:55:62 & 392 & 70 & 238 & 52:71:55:66 & 407 & 62 & 3 \\
\hline C499 & $140: 131: 114: 118$ & 854 & 104 & 315 & $125: 146: 109: 125$ & 856 & 87 & 5 \\
\hline C5315 & $460: 460: 520: 522$ & 3282 & 202 & 978 & $526: 520: 459: 460$ & 3288 & 197 & 25 \\
\hline C6288 & 645:674:695:842 & 5195 & 182 & 1130 & 691:847:644:674 & 5198 & 184 & 62 \\
\hline C7552 & 647:633:605:537 & 4105 & 66 & 1093 & 511:632:635:646 & 4114 & 44 & 37 \\
\hline $\mathrm{C} 880$ & 113:110:124:136 & 780 & 84 & 333 & $128: 130: 116: 113$ & 787 & 71 & 6 \\
\hline alu2 & 124:111:95:92 & 777 & 174 & 378 & 93:99:123:111 & 790 & 165 & 6 \\
\hline alu4 & 205:222:162:196 & 1470 & 278 & 587 & 201:156:197:231 & 1492 & 253 & 13 \\
\hline apex6 & $214: 261: 233: 200$ & 1417 & 75 & 478 & $209: 266: 233: 205$ & 1429 & 67 & 10 \\
\hline b9_n2 & $41: 47: 38: 32$ & 208 & 34 & 190 & $48: 48: 32: 34$ & 212 & 33 & 1 \\
\hline comp & $43: 48: 47: 46$ & 270 & 14 & 228 & $43: 48: 47: 46$ & 269 & 12 & 1 \\
\hline des & $879: 1075: 1035: 850$ & 6655 & 360 & 1878 & 986:1136:767:950 & 6656 & 277 & 96 \\
\hline duke2 & $95: 116: 96: 79$ & 676 & 159 & 333 & $80: 115: 78: 113$ & 693 & 142 & 5 \\
\hline $\mathrm{f} 5 \mathrm{~lm}$ & $35: 37: 30: 35$ & 244 & 70 & 188 & $36: 40: 37: 29$ & 252 & 67 & 2 \\
\hline $\operatorname{misex} 3$ & 111:132:138:157 & 990 & 189 & 425 & $163: 136: 132: 108$ & 1005 & 173 & 8 \\
\hline my_adder & $52: 54: 52: 54$ & 339 & 12 & 203 & $54: 52: 52: 54$ & 339 & 12 & 0 \\
\hline pcler8 & $40: 32: 28: 30$ & 174 & 20 & 143 & $26: 31: 40: 33$ & 174 & 20 & 0 \\
\hline rot & 232:208:190:194 & 1251 & 98 & 465 & 198:239:194:194 & 1254 & 91 & 10 \\
\hline sao2-hdl & $60: 75: 52: 63$ & 439 & 93 & 270 & 65:63:74:49 & 445 & 73 & 3 \\
\hline term 1 & $67: 56: 67: 82$ & 439 & 59 & 285 & $81: 71: 69: 57$ & 453 & 59 & 3 \\
\hline too_large & $769: 952: 907: 747$ & 6387 & 944 & 2895 & $935: 723: 752: 969$ & 6391 & 861 & 160 \\
\hline $\mathrm{tt} 2$ & $55: 66: 48: 58$ & 376 & 48 & 213 & $67: 56: 59: 49$ & 382 & 50 & 3 \\
\hline $\mathrm{x} 3$ & $191: 193: 236: 235$ & 1334 & 78 & 465 & 193:193:239:238 & 1369 & 70 & 11 \\
\hline $\begin{array}{c}\text { Total } \\
\text { Average }\end{array}$ & & 44170 & 4140 & & & $\begin{array}{c}44421 \\
+0.57 \%\end{array}$ & $\begin{array}{c}3668 \\
-11.4 \%\end{array}$ & \\
\hline
\end{tabular}

* (The result was picked from 250 runs of hMetis-Kway)

* (Total CPU times of 250 runs of hMetis-Kway)

cut size: the total number of pins required for all partitioned blocks

\section{CONCLUSION AND FUTURE WORK}

In this paper, a scheme coupling the graph-domain and logicdomain partitioners to explore a larger optimization flexibility of circuit partitioning is proposed. The scheme is shown to be very efficient in terms of CPU expenditure and is also quite capable of bringing further improvements on good partitioning produced by state-of-the-art partitioner hMetis-Kway. We conducted experiments on $29 \mathrm{MCNC}$ benchmark circuits for two- to four-way partitionings and obtained further cutsize reductions, from $12.3 \%$ to $11.1 \%$, than the high-quality results produced by hMetis-Kway. As logic-domain partitioner such as RAMBO, GBAW can be integrated with any newly developed powerful graph partitioner, this partitioning approach should be very practical and useful for various partitioning tasks.

\section{REFERENCES}

[1] Y. C. Wei and C. K. Cheng, "Ratio cut partitioning for hierarchical designs," IEEE Trans.Computer-Aided Design, vol. 10, pp. 911-921, July 1991.

[2] C. J. Alpert and A. B. Kahng, "Recent directions in netlist partitioning," Integr., VLSI J, vol. 19, no. 1-2, pp. 1-81, 1995.

[3] C. M. Fiduccia and R. M. Mattheyses, "A linear time heuristic for improving network partitions," in Proc. 19th ACM/IEEE Design Automation Conf., 1982, pp. 175-181.

[4] S. Dutt and W. Deng, "VLSI circuit partitioning by cluster-removal using iterative improvement techniques," in Proc. Int. Conf. Computer-Aided Design, 1996, pp. 194-200.
[5] C. W. Yeh, C. K. Cheng, and T. T. Y. Lin, "A probabilistic multicommodity-flow solution to circuit clustering problems," in Proc. Int. Conf. Computer-Aided Design, 1992, pp. 428-431.

[6] L. Hagen and A. B. Kahng, "Fast spectral methods for ratio cut partitioning and clustering," in Proc. Int. Conf. Computer-Aided Design, 1991, pp. 10-13.

[7] J. Y. Zien, M. D. F. Schlag, and P. K. Chan, "Multi-level spectral hypergraph partitioning with arbitrary vertex sizes," in Proc. Int. Conf. Computer-Aided Design, 1996, pp. 201-204.

[8] C. Kring and A. R. Newton, "A cell-replicating approach to mincutbased circuit partitioning," in Proc. Int. Conf. Computer-Aided Design, 1991, pp. 2-5.

[9] W.-K. Mak and D. F. Wong, "Minimum replication min-cut partitioning," in Proc. Int. Conf. Computer-Aided Design, 1996, pp. 205-210.

[10] M. Enos, S. Hauck, and M. Sarrafzadeh, "Replication for logic bipartitioning," in Proc. Int. Conf. Computer-Aided Design, 1997, pp. 342-349.

[11] H. H. Yang and D. F. Wong, "Optimal min-area min-cut replication in partitioned circuits," IEEE Trans. Computer-Aided Design, vol. 17, pp. 1175-1183, Nov. 1998.

[12] D. I. Cheng, C. C. Lin, and M. Marek-Sadowska, "Circuit partitioning with logic perturbation," in Proc. Int. Conf. Computer-Aided Design, 1995, pp. 650-655.

[13] M. Beardslee, B. Lin, and A. Sangiovanni-Vincentelli, "Communication based logic partitioning," in Proc. EURO-DAC '92, 1992, pp. 32-37.

[14] D. I. Cheng, S. C. Chang, and M. Marek-Sadowska, "Parititioning combinational circuits in graph and logic domains," in Proc. SASIMI-93, 1993, pp. 404-412.

[15] R. Kuznar, F. Brglez, and B. Zajc, "Multi-way netlist partitioning into heterogeneous FPGA's and minimization of total device cost and interconnect," in Proc. 31th ACM/IEEE Design Automation Conf., 1994, pp. 238-243.

[16] B. Hendrickson and R. Leland, "A multilevel algorithm for partitioning graphs," Sandia Nat. Labs., Albuquerque, NM, Tech. Rep. SAND931301, 1993. 
[17] _ - "The Chaco user's guide," Sandia Nat. Labs, Albuquerque, NM, Tech. Rep. SAND93-2339, 1993.

[18] G. Karypis and V. Kumar, "Multilevel graph partitioning schemes," in Proc. 1995 Int. Symp. Physical Design, 1995, pp. 113-122.

[19] G. Karypis, R. Aggarwal, V. Kumar, and S. Shekhar, "Multilevel hypergraph partitioning: Application in VLSI domain," in Proc. 34th ACM/IEEE Design Automation Conf., 1997, pp. 526-529.

[20] C. J. Alpert, J.-H. Huang, and A. B. Kahng, "Multilevel circuit partitioning," in Proc. 34th ACM/IEEE Design Automation Conf., 1997, pp. 530-533.

[21] S. Wichlund and E. J. Aas, "On multilevel circuit partitioning," in Proc. Int. Conf. Computer-Aided Design, 1998, pp. 505-511.

[22] G. Karypis and V. Kumar, "Multilevel $k$-way hypergraph partitioning," in Proc. 36th ACM/IEEE Design Automation Conf., 1999, pp. 343-348.

[23] A. E. Caldwell, A. B. Kahng, and I. L. Markov, "Improved algorithms for hypergraph bipartitioning," in Proc. Asia South Pacific Design Automation Conf., 2000, pp. 661-666.

[24] J. Cong and S. K. Lim, "Edge separability based circuit clustering with application to circuit partitioning," in Proc. Asia South Pacific Design Automation Conf., 2000, pp. 429-434.

[25] —_, "Performance driven multiway partitioning," in Proc. Asia South Pacific Design Automation Conf., 2000, pp. 441-446.

[26] M. Wang, S. K. Lim, J. Cong, and M. Sarrafzadeh, "Multi-way partitioning using bi-partition heuristics," in Proc. Asia South Pacific Design Automation Conf., 2000, pp. 667-672.

[27] K.-T. Cheng and L. A. Entrena, "Multi-level logic optimization by redundancy addition and removal," in Proc. EDAC-93, Feb. 1993, pp. 373-377.

[28] S. C. Chang, M. Marek-Sadowska, and K. T. Cheng, "Perturb and simplify: Multilevel boolean network optimizer," IEEE Trans. ComputerAided Design, vol. 15, pp. 1494-1504, Dec. 1996.

[29] S. C. Chang, K. T. Cheng, N. S. Woo, and M. Marek-Sadowska, "Layout driven logic synthesis for FPGA," in Proc. 31th ACM/IEEE Design Automation Conf., June 1994, pp. 308-313.

[30] S.-C. Chang, K.-T. Cheng, N.-S. Woo, and M.-S. M, "Postlayout logic restructuring using alternative wires," IEEE Trans. Computer-Aided Design, vol. 16, pp. 587-596, June 1997.

[31] S. C. Chang and M. Marek-Sadowska, "Perturb and simplify: Multilevel boolean network optimizer," in Proc. Int. Conf. Computer-Aided Design, Nov. 1994, pp. 2-4.

[32] L. A. Entrena and K.-T. Cheng, "Sequential logic optimization by redundancy addition and removal," in Proc. Int. Conf. Computer-Aided Design, Nov. 1993, pp. 310-315.

[33] — - "Combinational and sequential logic optimization by redundancy addition and removal," IEEE Trans. Computer-Aided Design, vol. 14, pp. 909-916, July 1995.

[34] L. Entrena, J. Espejo, E. Olias, and J. Uceda, "Timing optimization by an improved redundancy addition and removal technique," in Proc. 1996 Design Automation Conf., 1996, pp. 342-347.

[35] S. C. Chang, L. P. V. Ginneken, and M. Marek-Sadowska, "Fast boolean optimization by rewiring," in Proc. Int. Conf. Computer-Aided Design, 1996, pp. 262-269.

[36] C.-W. Chang and M. Marek-Sadowska, "Single-pass redundancy addition and removal," in Proc. IEEE/ACM Int. Conf. Computer-Aided Design, 2001, pp. 606-609.

[37] E. M. Sentovich et al., "SIS: A system for sequential circuit synthesis," in Proc. ERL Memorandum UCB/ERL, vol. M92/41, 1992.

[38] Y.-L. Wu, W. Long, and H. Fan, "A fast graph-based alternative wiring scheme for boolean networks," in Proc. IEEE/ACM Int. VLSI Design, 2000, pp. 268-273.

[39] Y. L. Wu, C. N. Sze, C. C. Cheung, and H. Fan, "On improved graph-based alternative wiring scheme for multi-level logic optimization," in Proc. IEEE Int. Conf. Electron. Circuits Syst. (ICECS), 2000, pp. 654-657.

[40] Y. L. Wu, X. L. Yuan, and D. I. Cheng, "Circuit partitioning with coupled logic restructuring techniques," in Proc. Asia South Pacific Design Automation Conf., 2000, pp. 655-660.

[41] C. J. Alpert, J.-H. Huang, and A. B. Kahng, "The ISPD98 circuit benchmark suite," in Proc. Int. Symp. Physical Design, 1997, pp. 80-85.

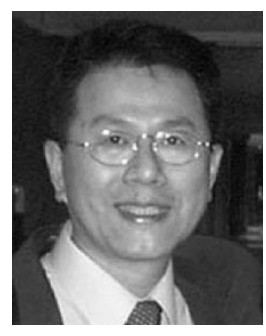

Yu-Liang Wu (M'96) received the B.S. and M.S. degrees in computer science from Florida International University, Miami, FL, in 1983 and 1984 respectively, and the Ph.D. degree in electrical and computer engineering from the University of California Santa Barbara, in 1994.

In 1985, he worked with the Internet Systems Corporation as a System Programmer on network communication protocols (DARPA TCP/IP, Telnet). From 1986 to 1988, he worked with AT\&T Bell Labs on the development of several telephone distributed operation systems (RMAS, MFOS). From 1988 to 1989, he worked for Amdahl Corporation on tester software designs. From 1994 to 1996, he was with Cadence Design Systems Incorporation as a senior MTS where he worked in research and development of the silicon synthesis product (PBS), targeting at binding the gap between logical and physical level optimizations for deep-submicron chip designs. In 1996, he joined The Chinese University of Hong Kong. His current research interests include optimization of logic and physical design automation of very large scale integration (VLSI) circuits and field programmable gate arrays (FPGAs) related computer-aided design (CAD) tool designs and architectural analysis/optimization.

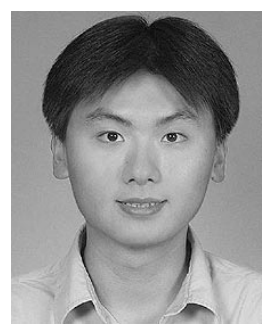

Chak-Chung Cheung received the B.Eng. and M.Phil. degree in computer engineering and computer science and engineering from The Chinese University of Hong Kong (CUHK), in 1999 and 2001, respectively.

In 2001, he worked as a System Administrator with the Center of Large-Scale Computation (CLC), Cluster Technology, Hong Kong. He is now an Instructor with the Deparment of Computer Science and Engineering, CUHK, and is responsible for the courses on Internet and Web Programming Technologies. His current research interests include optimization of logic and physical design automation of very large scale integration (VLSI) ASIC/FPGA designs and high-level synthesis of reconfigurable computing.

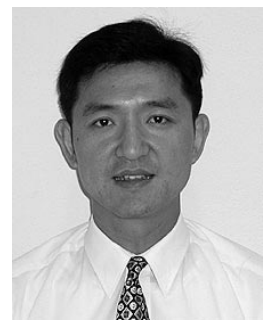

David Ihsin Cheng received the B.S. degree in computer engineering from National Chiao-Tung University, Hsinchu, Taiwan, in 1986, the M.S. degree in computer science and the $\mathrm{Ph} . \mathrm{D}$. degree in computer engineering, both from the University of California, Santa Barbara, in 1990 and 1995, respectively.

He is currently with Faraday Technology Corp. Sunnyvale, CA, where he specializes in ASIC implementation. He was previously with Ultima Interconnect, Sunnyvale, CA, Mentor Graphics, Wilsonville, OR, IBM Watson Research Center, Yorktown Heights, NY and AT\&T Bell Labs. His research interests include all aspects of the logical and physical implementations of ASICs.

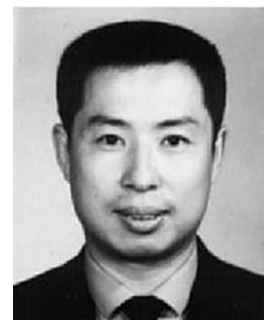

Hongbing Fan received the B.S. degree in mathematics and the Ph.D. degree in operational research and control theory from Shandong University, Jinan, China, P.R.C., in 1982 and 1990, respectively, and received the second Ph.D. degree in Computer Science from the University of Victoria, Canada, in 2003.

In 1990, he joined the mathematics department at Shandong University as an Associate Professor. He worked with the Department of Computer Science and Engineering at The Chinese University of Hong Kong (CUHK), as an Research Associate in 1998. His research interests include combinatorial algorithm and complexity, graph theory, and various topics in very large scale integraton (VLSI) design and operations research. 